\title{
Kein Menschenrecht auf ärztliche Suizidhilfe
}

René Raggenbass ${ }^{a}$,

Hanspeter Kuhn ${ }^{b}$

a Dr. med., Mitglied des Zentralvorstandes der FMH

b Fürsprecher, stv. Generalsekretär der FMH
Korrespondenz:

Hanspeter Kuhn FMH

Postfach 170

CH-3000 Bern 15

Tel. 0313591111

Fax 0313591112

lex@fmh.ch
Der Bundesgerichtsentscheid wurde in der Tagespresse im Februar 2007 vorgestellt. Teilweise entstand der Eindruck, das Bundesgericht habe ein Recht auf ärztliche Hilfe beim Suizid eingeführt. Dies trifft nicht zu.

\section{Der Fall}

«X. Y. (geb. 1953) leidet an einer schweren bipolaren affektiven Störung. Er beging zwei Selbstmordversuche und wurde wiederholt stationär behandelt. Am 1. Juli 2004 trat er dem Verein 〈Dignitas〉 bei und bat diesen am 8 . November 2004, für ihn eine Freitodbegleitung in die Wege zu leiten, da er sein Leben aufgrund der nur schwer behandelbaren Krankheit als nicht mehr menschenwürdig erachte. Da für die hierzu erforderlichen 15 Gramm Natrium-Pentobarbital kein ärztliches Rezept erhältlich gemacht werden konnte, gelangte er am 8. Juni 2005 mit dem Antrag an mehrere Instanzen, es sei ihm zu bewilligen, über den Verein 〈Dignitas〉 ohne Vorlage einer ärztlichen Verschreibung 15 Gramm dieses Stoffes zu beziehen. Er begründete sein Ersuchen jeweils damit, dass er als handlungsfähiger Mensch einen Anspruch darauf habe, sein eigenes Leben risiko- und schmerzfrei sowie ohne Gefahren für Dritte beenden zu können.» (Sachverhaltswiedergabe im Urteil, A).

Das Bundesgericht hat aufgrund der folgenden Erwägungen die Beschwerde von X.Y. abgewiesen [Auszug, kursive Hervorhebungen HP. Kuhn]:

Die Ziele der EMRK können nicht nur mit Natrium-Pentobarbital erreicht werden

4.1.2 «Für Betäubungsmittel gilt das Heilmittelgesetz, soweit sie als Heilmittel verwendet werden (Art. 2 Abs. 1 lit. b HMG); wenn dieses keine oder eine weniger weit gehende Regelung trifft, ist das Betäubungsmittelgesetz anwendbar (Art. 2 Abs. 1 bis BetmG).»

6.2.3 « Für eine wirksame Umsetzung der in Art. 8 Ziff. 1 EMRK verankerten Freiheit, über die Beendigung des eigenen Lebens entscheiden zu können, ist eine vorbehaltlose Abgabe von Natrium-Pentobarbital nicht erforderlich, auch wenn es sich für den Suizid offenbar besonders gut eignen soll.»

\section{Kein Arzt muss Suizidhilfe leisten - aber «ärztliche Suizidhilfe ist nicht mehr ausgeschlossen»}

Das Bundesgericht stellt in der Erwägung 6.2.4 die revidierte Richtlinie der SAMW zur Betreuung der Patientinnen und Patienten am Lebensende vor und schliesst daraus: «Entgegen den Einwendungen des Beschwerdeführers kann somit im Rahmen der anerkannten medizinischen Berufsregeln durchaus ein ärztliches Rezept für die Abgabe von Natrium-Pentobarbital ausgestellt werden, falls im Einzelfall die entsprechenden Voraussetzungen erfüllt sind. Wie das Bundesgericht bereits festgestellt hat, ist diesbezüglich heute ein Umdenken in dem Sinne im Gang, dass die Suizidhilfe zusehends als freiwillige ärztliche Aufgabe verstanden wird, die zwar keinem Arzt aufgedrängt werden kann, aber auch aufsichts- bzw. standesrechtlich nicht ausgeschlossen erscheint, solange bei der Untersuchung, Diagnose und Abgabe die ärztlichen Sorgfaltspflichten eingehalten werden (Urteil 2P.310/2004 vom 18. Mai 2005, E. 4.3 mit Hinweisen) bzw. sich der betroffene Arzt nicht hauptsächlich vom Sterbewunsch seines Patienten leiten lässt, ohne dessen Entscheid nach wissenschaftlichen Gesichtspunkten gründlich auf seine medizinische Begründetheit hin zu überprüfen (so das Urteil des Verwaltungsgerichts des Kantons Zürich vom 15. Juli 1999, E. 4e, publ. in: ZBI 101/2000 S. 489 ff.).»

\section{Suizidhilfe bei psychisch Kranken? - nur «mit äusserster Zurückhaltung»}

Zur Suizidbeihilfe bei psychisch Kranken hält das Urteil fest: «Nach neueren ethischen, rechtlichen und medizinischen Stellungnahmen ist auch in solchen Fällen eine allfällige Verschreibung von Natrium-Pentobarbital nicht mehr notwendigerweise kontraindiziert und generell als Verletzung der medizinischen Sorgfaltspflichten ausgeschlossen (Rippe/Schwarzenegger/Bosshard/ Kiesewetter, Urteilsfähigkeit von Menschen mit psychischen Störungen und Suizidbeihilfe, in: SJZ 101/2005 S. 53ff., dort S. 90; Empfehlung 
Nr. 6 vom 27. April 2005 der Nationalen Ethikkommission im Bereich der Humanmedizin betreffend 〈Beihilfe zum Suizid»). Doch ist dabei äusserste Zurückhaltung geboten: Es gilt zwischen dem Sterbewunsch zu unterscheiden, der Ausdruck einer therapierbaren psychischen Störung ist und nach Behandlung ruft, und jenem, der auf einem selbst bestimmten, wohlerwogenen und dauerhaften Entscheid einer urteilsfähigen Person beruht (‘Bilanzsuizid〉), den es gegebenenfalls zu respektieren gilt. Basiert der Sterbewunsch auf einem autonomen, die Gesamtsituation erfassenden Entscheid, darf unter Umständen auch psychisch Kranken NatriumPentobarbital verschrieben und dadurch Suizidbeihilfe gewährt werden (vgl. Rouiller/Roussianos, a.a.O., Rz. 45; Petermann, a.a.O., Entwurf Suizidpräventionsgesetz, S. 1117-1123).»

\section{Natrium-Pentobarbital bleibt rezeptpflichtig}

Um diese Voraussetzungen zu prüfen, müsste gemäss Bundesgericht ein «vertieftes psychiatrisches Fachgutachten» erstellt werden. Um eine solche Begutachtung sicherzustellen, muss gemäss Bundesgericht an der ärztlichen Verschreibungspflicht des Medikaments festgehalten werden. Die Verantwortung kann gemäss Urteil «nicht (allein) in die Hände der Sterbehilfeorganisationen gelegt» werden. «Deren Aktivitäten haben verschiedentlich zu Kritik Anlass gegeben; so hat etwa eine Basler Studie aufgrund einer Analyse von 43 Fällen von durch «Exit assistierten Suiziden zwischen 1992 und 1997 gerade die mangelnde Berücksichtigung psychiatrischer oder sozialer Faktoren beim Suizidentscheid beanstandet (Frei/Schenker/Finzen/ Kräuchi/Dittmann/Hoffmann-Richter. Assisted suicide as conducted by a «Right-to-Die>-society in Switzerland: A descriptive analysis of 43 consecutive cases. Swiss Med Wkly. 2001;131[25/26]: 375-80). Es kann deshalb nicht gesagt werden, dass durch die Abgabe des Natrium-Pentobarbitals und die Delegation der Verantwortung für dessen Einsatz an eine Sterbehilfeorganisation der Schutzzweck eben so gut erfüllt würde wie durch das Festhalten an der ärztlichen Rezeptpflicht.»

\section{Kommentar}

Das Bundesgerichtsurteil ist aus unserer Sicht ausgewogen. Es bestätigt grundsätzlich den in der revidierten SAMW-Richtlinie zur Sterbehilfe aufgezeigten Weg.
Gleichzeitig stellen wir fest, dass das Urteil nicht unterscheidet zwischen Suizidhilfe und Sterbehilfe. Diese beiden Begriffe sind grundverschieden, und sie betreffen nicht dieselbe klinische Realität:

- Sterbehilfe betrifft definitionsgemäss eine Person, dessen Tod infolge einer nicht behandelbaren und tödlichen Krankheit unvermeidlich bevorsteht;

- Suizidhilfe hingegen betrifft Personen, deren Tod nicht unabwendbar bevorsteht.

Dieser Unterschied kann zu erheblichen Missverständnissen führen, insbesondere wenn es um den Bereich der psychischen Leiden geht. Auch wenn ein psychisches Leiden eine Person in den Suizid treiben kann, führt die psychische Krankheit als solche, egal wie schwer sie ist, nicht zum Tod.

Was die Suizidbeihilfe bei psychisch Kranken angeht, ist unseres Erachtens aus ärztlicher Sicht noch mehr als die vom Bundesgericht verlangte «äusserste Zurückhaltung» geboten - nämlich der Verzicht auf ärztliche Suizidhilfe für psychisch Kranke. Dies aus den folgenden Gründen: Der Geltungsbereich der SAMW-Richtlinie ist unseres Erachtens zu Recht - eng gefasst. Eine der Voraussetzungen gemäss Richtlinie ist: «Die Erkrankung des Patienten rechtfertigt die Annahme, dass das Lebensende nahe ist.» Auch wenn psychisches Leiden subjektiv als unerträglich erlebt werden kann, folgt es anderen Gesetzmässigkeiten als somatisches Leiden am Lebensende - psychisches Leiden ist von Krisen- und Erholungsphasen gekennzeichnet. Das Erleben der Krise und des Leidens hängt sehr stark von subjektiven Persönlichkeitsfaktoren ab und ist zudem multifaktoriell bestimmt. Viele dieser Faktoren sind heute wissenschaftlich noch ungenügend verstanden. Würde der Weg zur Suizidbeihilfe und/oder zur Sterbehilfe bei psychisch Kranken generell und im besonderen beim konkret vorliegenden Fall eröffnet, hiesse dies, die Komplexität des psychischen Leidens in willkürlicher und unwissenschaftlicher Art und Weise über einen Leisten zu schlagen.

Bis zum Vorliegen neuer Erkenntnisse spricht sich der Zentralvorstand der FMH deshalb klar gegen die Suizidbeihilfe bei psychisch Kranken aus. 